http://jmscr.igmpublication.org/home/

ISSN (e)-2347-176x ISSN (p) 2455-0450

crossref DOI: https://dx.doi.org/10.18535/jmscr/v7i11.47

Journal Of Medical Science And Clinical Research

\title{
Evaluation of Hepatic changes in patients of Metabolic Syndrome
}

\author{
Authors \\ Dr Vinay Kumar ${ }^{1 *}$, Dr Shivendra Verma², Dr Mohit Sachan ${ }^{3}$, \\ Dr Mahendra Pal Singh ${ }^{4}$, Dr Richa Giri ${ }^{5}$ \\ ${ }^{1}$ Associate Professor, Dept. of Medicine GSVM Medical College, Kanpur \\ ${ }^{2,3,4}$ Assistant Professor, Dept. of Medicine GSVM Medical College, Kanpur \\ ${ }^{5}$ Professor and Head, Dept. of Medicine GSVM Medical College, Kanpur \\ *Corresponding Author
}

Dr Vinay Kumar

Associate Professor, Dept. of Medicine, GSVM Medical College, Kanpur, 208002, India

\begin{abstract}
Background: The metabolic syndrome is a constellation of clinical and metabolic abnormalities including abdominal obesity, hypertension, dyslipidernia and impaired fasting glucoselor impaired glucose tolerance. The accumulation of excessive fat in the liver is a manifestation of lipotoxicity to the liver. Initially this is characterized by only steatosis. but later on free fatty acids induce inflammatory reactions called as steatohepatits. The long-term consequences of non alcoholic steatohepatitis (NASH) include cirrhosis of the liver and hepatocellular carcinoma. In this study we studied the hepatic manifestations of metabolic syndrome.

Methods: The study was carried out in the Post-graduate Department of Medicine, S.N. Medical College, Agra in the period of may 2007 to September 2009. The material of the study included 30 cases of metabolic syndrome selected from the medicine outdoor clinic (including diabetic clinic) and medicine indoor wards as well as from GI clinic. Data were collected regarding antropometric and clinical parameters. Symptoms and signs related to liver involvement were assessed. Laboratory parameters and imaging study were done to look for hepatic involvement and also to exclude other causes of hepatic involvement. In selected cases liver biopsy was done.

Results: Study group consisted of 12 male (40\%) and 18 (60\%) female patients. Most of the patients (60\%) of metabolic syndrome were asymptomatic. Fatigue/malaise was present in 11 (36.7\%) of patients, anorexia in 8 (26.7\%) and right upper quadrant discomfort in $6(20 \%)$ of patients. Hepatomegaly was present in 7 (23.3\%) patients. Ascites was present in $4(13.3 \%)$ patients, Icterus was present in $3(10 \%)$ patients while $2(6.7 \%)$ of patients have hemetemesis/malena.10 out of 30 patients (33.3\%) have increased hepatic echogenicity on USG abdomen while 20 (66.7\%) patients have normal sonographic study liver of Histopathological evaluation of liver was done in 7 male and 7 female patients. 3 out of 14 patients (21.4\%) have normal histopathology of liver, while 4 patients $(28.6 \%)$ have grade-I changes, 4 patients $(28.6 \%)$ have grade-II and only one patient (7.1) have grade III histopathological changes in liver. 2(14.3\%) patients showed grade IV histopathological changes. These changes were statistically significant (P. value $<0.05$ ).

Conclusion: Most of the patients of metabolic syndrome (60\%) had no symptoms or signs of liver disease. Persistent fatigue/malaise (36.7\%) and anorexia (26.7\%) were most common symptoms observed. Hepatomegaly was prevalent in $23.3 \%$ of the cases.There was poor correlation between abnormal liver function tests and ultrasonographic abnormalities of liver. Only $53.3 \%$ patients of metabolic syndrome with abnormal liver function tests were found to have abnormal ultrasonographic findings. Positive correlation between duration of diabetes mellitus, obesity, hepatomegaly, liver function tests and ultrasonographic findings were observed.
\end{abstract}




\section{Introduction}

The term metabolic syndrome has its origin in 1923 when Kylin $\mathbf{E}^{\mathbf{1}}$ describe a syndrome involving hypertension, hyperglycemia and hyperuricaemia. In 1989, Kaplan ${ }^{2}$ renamed the syndrome 'The Deadly Quarlet' and in 1992 it was again renamed the 'insulin resistance syndrome.

The metabolic syndrome is a constellation of clinical and metabolic abnormalities including abdominal obesity, hypertension, dyslipidernia and impaired fasting glucose/or impaired glucose tolerance. All these manifestations are surrogate marker of insulin resistance which is the crux abnormality associated with metabolic syndrome. Visceral fat is more metabolically active because of its rich nerve (b3) and blood supply and hence associated with increased release of free fatty acids (FFA's) which are diverted to the nonadipose tissue including liver and muscle ${ }^{3,4}$. The increased delivery of FFA's to liver is partially due to the decreased disposal to adipose tissue because of inactivity of lipoprotein lipase (LPL), an insulin sensitive enzyme, and direct access of FFA's to liver through portal vein. The long-term consequences of non alcoholic steatohepatitis (NASH) include cirrhosis of the liver and hepatocellular carcinoma.

Patients with simple steatosis have a relatively benign liver disease with a risk of developing clinical evidence of cirrhosis over 15-20 years in the order of 1-2 Patients with NASH and fibrosis can progress to cirrhosis, defined histologically or clinically with the risk varying from $0 \%$ at 5 years to $12 \%$ over 8 years. Once cirrhosis develops, patients are at higher risk of developing hepatic decompensation and of dying from liver related cause including hepatocellular carcinoma.

Therefore, NASH has to be identified early in the disease course and requires appropriate therapy to prevent long-term undesirable consequences.

Patients: The study was carried out in the Postgraduate Department of Medicine, S.N. Medical College, Agra in the period of may 2007 to September 2008.
The material of the study included 30 cases of metabolic syndrome selected from the medicine outdoor clinic (including diabetic clinic) and medicine indoor wards as well as from GI clinic.

Diagnosis of metabolic syndrome was made according National Cholesterol Education Programme Adult Treatment Panel III (ATP III) criteria. ${ }^{5,6,7}$ Study included patients who have at least three of the following criteria.

Elevated waist circumference: $\geq 90 \mathrm{~cm}$ for men and $80 \mathrm{~cm}$ for women

Elevated Triglycerides: $\geq 150 \mathrm{mg} / \mathrm{dl}$

Reduced HDL (gold) cholesterol: $\leq 40 \mathrm{mg} / \mathrm{dl}$ for men $50 \mathrm{mg} / \mathrm{dl}$ for women

Elevated blood pressure: $\geq 130 / 85 \mathrm{mmHg}$

Elevated fasting glucose: $\geq 100 \mathrm{mg} / \mathrm{dl}$

Patients with history of alcohol intake > 20 gm/day (women), > 30 gm/day (men) were excluded from the study.

Chronic liver disease due to other causes i.e. hepatitis B, hepatitis C, drug induced and autoimmune disease, decompensated cirrhosis (Child Pugh-3), patient with overt cardiac dysfunction, recent stroke and pregnancy were also excluded for relevant reasons.

Patients on following drugs were excluded from this study:

- Antiarrhythmic- Amiodarone

- Antibiotic- Tetracycline (high dose), Isoniazid, Rifampcin, Pyrazinamide.

- AnticonvulsantPhenytoin carbamazepine, Valproic acid.

- Antiviral-Zidovudine, Protease inhibitors (eg. Indinavir, Ritonavir)

- Antifungal- Ketoconazole, Fluconazole, Itraconazole.

- Antiandrogen- Flutamide.

- Antidepressant-

Amitriptyline, Venlafaxine, Fluoxetine, Duloxitine, Sertraline.

- Antihypertensive- Methyldopa

- Antipsychotic- Risoperidone

- Diuretics-Chlorothiazide

- Oral hypoglycemic-Acorbose

- Oral contraceptive 
- Patients with history suggestive of inflammatory bowel disease, jejunoileal bypass and total parentralnutrition were also excluded from this study.

\section{Methods}

The enrolled patients were subjected to a protocol which included detailed history regarding mode of onset, presentation, duration of illness, personal antecedents including history of alcohol intake. The subjects were subjected to thorough general physical examination.

Clinical Profile and Measurements: The data of blood pressure measurement and anthropometric parameters (waist circumference) were collected for all subjects.

Blood pressure was measured in the right arm with the subject seated and rested for 5 minutes using a standard mercury sphygmonometer. At least two reading at 5 minute intervals were taken and if an abnormal value was obtained, then another reading was taken after 30 minutes of rest. Waist circumference was measured midway between the iliac crest and the lower most margin of the ribs with bare belly and at the end of normal expiration using the following approach (Grundy, Cleeman and Daniel, 2005)

-Locate the top of right iliac crest.

- Place a measuring tape in the horizontal plane around the abdomen, at the level of the iliac crest.

- The tape should be parallel with the floor and should not compress the skin.

- Take the measurement at the end of normal expiration.

Evaluation of hepatic manifestation in patients of metabolic syndrome

Symptoms

- Loss of appetite (anorexia)

- Fatigue

- Malaise

- Right upper quadrant discomfort

- Upper GI bleeding (hemetemesis/malena)

- Abdominal distension
Signs

- Hepatomegaly

- Splenomegaly

- Signs of portal hypertension.

All patients selected were subjected to following laboratory investigations.

- Complete haemogram

- Blood sugar (fasting and post prandial)

- Renal function test

- Urine routine and microscopic examination

- Liver function tests (Serum bilirubin, SGOT/SGPT, PT, S. proteins)

- Lipid profile

- Electrolyte $\left(\mathrm{Na}^{+}, \mathrm{K}^{+}, \mathrm{iCa}^{++}\right)$

- USG abdomen specially for size, shape and echotexture of liver and to exclude other causes of chronic liver disease.

Patients those with abnormal liver function tests were subjected to further investigations.

1) Investigations to rule out other causes responsible for abnormal LFT.

- Serological markers for HBV, HCV

- Autoimmune marker

2) The selected patients were subjected for liver biopsy to find out histopathological changes in NAFLD and NASH and to exclude other causes of chronic liver disease.

The histopathological changes in NASH include hepatic steatosis, ballooning degeneration, acute, chronic or mixed inflammation, perisinusoidal fibrosis, and Mallory hyaline bodies.

Burnt et al $^{8}$ have described fibrotic changes in NASH in 4 stages.

\section{Stages of NASH}

Stage I: Zone III perisinusoidal or pericellular fibrosis, either focal or diffuse

Stage II: Stage I + extensive periportal fibrosis

Stage III: Stage II + focal or extensive bridging fibrosis

Stage IV: Cirrhosis of liver

Statistical Analysis: Categorical and continuous data were presented as proportion, median and 
range or mean, and standard deviation depending on the distribution. Categorical variables were analyzed using the Chi-squared test with Yate's correctionas applicable. Continuous parametric and nonparametric data were analyzed by unpaired $\mathrm{t}$ and Mann-Whitney $\mathrm{U}$ tests, respectively. $\mathrm{P}$ values less than 0.05 were considered significant.

\section{Results}

Patient Characteristics: Study group consisted of 12 male (40\%) and $18(60 \%)$ female patients. Majority of patients $(40.0 \%)$ belong to age group 40-49 years .Mean age of all the patients was $47.6 \pm 7.5$ years. The mean age of males and females was $49.6 \pm 8.0$ and $46.2 \pm 7.1$ respectively. The prevalence of component of metabolic syndrome in men \& women were, central obesity in $9(75 \%)$ and $15(83.3 \%)$ patients respectively, low HDL cholesterol in $6(50 \%)$ and $14(77.8 \%)$ patients respectively, high triglycerides in 9 $(75 \%)$ and $12(66.7 \%)$ respectively, impaired fasting glucose $(>100 \mathrm{mg} / \mathrm{dl})$ or diabetes in 6 $(50.0 \%)$ and $6(33.3 \%)$ respectively and elevated blood pressure in $9(75 \%)$ of men and $16(88.9 \%)$ of women.

Out of 6 diabetic male patients $4(66 \%)$ and out of 6 diabetic female patients $1(16.7 \%)$ were known diabetic for more than 10 years. Out of 9 hypertensive male patients $2(22 \%)$ and out of 7 hypertensive female patients $2(28.6 \%)$ were known diabetic for more than 10 years.

Out of 9 male patients of metabolic syndrome who have waist circumference equal to more than 90 $\mathrm{cm}, 4$ patients $(44.5 \%)$ have waist circumference between 90-99 cm, 3 (33.3\%), have waist circumference between $100-109 \mathrm{~cm}$ and $2(22.2 \%)$ patients have waist circumference more than 110 $\mathrm{cm}$. Mean waist circumference of males was $97.9 \pm 7.2 \mathrm{~cm}$. Out of 15 female patients who have waist circumference equal to or more than $80 \mathrm{~cm}$ $3(20 \%)$ patients have between 80-89\%, $6(40 \%)$ have between $90-99 \%$ and $6(40 \%)$ have waist circumference more than $100 \mathrm{~cm}$. Mean waist circumference of females was $97.8 \pm 2.1 \mathrm{~cm}$.
Nine male patients have elevated triglycerides level. Out of 9 male patients of metabolic syndrome $6(66.7 \%)$ patients have triglyceride level between 150-174 mg\%, 2 patients (22.2\%) have triglyceride level between $175-199 \mathrm{mg} \%$ and only one patient $(11.1 \%)$ have triglyceride level more than $200 \mathrm{mg} \%$. Mean triglycerides level of males was $160.1 \pm 22.6 \mathrm{mg} \%$. Out of 12 female patients of metabolic syndrome $7(58.3 \%)$ have triglyceride level between 150-174 mg\%, 3 (25\%) have triglyceride level between 175-199 $\mathrm{mg} \%$ and $3(16.7 \%)$ patients have triglyceride level more than $200 \mathrm{mg} \%$. Mean triglycerides level of females was $162.7 \pm 27.2 \mathrm{mg} \%$.

Out of 6 male patients of metabolic syndrome who have HDL level <40 mg/dl 5 patients $(83.3 \%)$ have HDL level between 30-39 mg/dl, 1 (16.7\%) patient has HDL level less than $30 \mathrm{mg} / \mathrm{dl}$. Mean HDL level of males was $40.8 \pm 6.4 \mathrm{mg} / \mathrm{dl}$. Out of 14 female patients who have HDL level <50 $\mathrm{mg} / \mathrm{dl}, \quad 6(42.9 \%)$ patients have HDL level between $40-49 \mathrm{mg} / \mathrm{dl}, 7$ (50\%) patients have between 30-39 and only 1 (7.1\%) patient have HDL level less than $30 \mathrm{mg} / \mathrm{dl}$. Mean level of HDL of females was $43.4 \pm 7.5 \mathrm{mg} / \mathrm{dl}$.

\section{Clinical Assessment}

Most of the patients (60\%) of metabolic syndrome were asymptomatic. Fatigue/malaise was present in $11(36.7 \%)$ of patients, anorexia in $8(26.7 \%)$ and right upper quadrant discomfort in $6(20 \%)$ of patients.

Most of the patients of metabolic syndrome have no sign suggestive of hepatic involvement. Hepatomegaly was present in 7 (23.3\%) patients. Ascites was present in $4(13.3 \%)$ patients, Icterus was present in $3(10 \%)$ patients while $2(6.7 \%)$ of patients have hematemesis and or melena.

\section{Laboratory Evaluation}

S.Bilirubin was elevated in $8(26.7 \%)$ of patients of metabolic syndrome. This elevation was insignificant (p. Value >0.05).ALT was significantly (p. Value <0.05) elevated in $14(46.7 \%)$ patients. AST was insignificantly (p. 
value $>0.05)$ elevated in $12(56.7 \%)$ patients. $\mathrm{S}$. Alkaline Phosphatase was abnormal in 12(40.0\%) of patients. S. albumin was decreased in $10(33.3 \%)$ patients of metabolic syndrome. These changes were statistically significant (p.value $<0.05$ ).

\section{Ultrasonographic Changes}

10 out of 30 patients (33.3\%) have increased hepatic echogenicity on USG abdomen while 20 (66.7\%) patients have normal sonographic study of liver. These changes were statistically insignificant ( $p$. value $>0.05$ ).

\section{Histological changes}

Histopathological evaluation of liver was done in 7 male and 7 female patients. 3 out of 14 patients (21.4\%) have normal histopathology of liver, while 4 patients $(28.6 \%)$ have grade-I changes, 4 patients $(28.6 \%)$ have grade-II and only one patient (7.1) have grade III histopathological changes in liver. 2(14.3\%) patients showed grade IV histopathological changes. These changes were statistically significant $(P$. value<0.05).Liver biopsy was done in 14 patients with abnormal liver function test. Out of these 14 patients 4 (28.6\%) had normal liver biopsy while 10 (71.4\%) had abnormal liver histology. 13 obese patients were undergone liver biopsy, results were normal in $3(23 \%)$ patients while $10(76.9 \%)$ had normal liver histology. Liver biopsy was done in 11 patients with high triglyceride level 8 (72.9\%) patients had abnormal liver histology while normalcy was maintained in $3(27.2 \%)$ patients. 8 diabetic patients were undergone liver histological examination, all the patients (100\%) have abnormalechotexture. 7 patients with abnormal liver ultrasnography were also evaluated for liver histology. 2 (28.6\%) patients had normal liver histology while $5(71.4 \%)$ patients were with abnormal histological findings.

\section{Discussion}

The present study was undertaken to find out prevalence of hepatic involvement and their clinical presentation in the patients of metabolic syndrome and also to determine high risk patients who have potentiality for NAFLD.

The present study concludes that

1. Most of the patients of metabolic syndrome $(60 \%)$ had no symptoms or signs of liver disease. Persistent fatigue/malaise $(36.7 \%)$ and anorexia (26.7\%) were most common symptoms observed. Hepatomegaly was prevalent in $23.3 \%$ of the cases.

2. There was poor correlation between abnormal liver function tests and ultrasonographic abnormalities of liver. Only $53.3 \%$ patients of metabolic syndrome with abnormal liver function tests were found to have abnormal ultrasonographic findings.

3. Patients with hepatomegaly, abnormal liver function tests and abnormal ultrasonography were at higher risk and had potential for NAFLD.

4. Positive correlation between duration of diabetes mellitus, obesity, hepatomegaly, liver function tests and ultrasonographic findings were observed.

Females are more frequently affected by NAFLD. NAFLD was believed to occur primarily in middle aged obese women (Angulo P. et al) ${ }^{9}$. More recent studies have shown that men and women equally affected by it, in addition even higher involvement of men has also been noted (Diehl Am et. al $)^{10}$. In our study males were more affected with NAFLD than females. (Flack yatter. et al $)^{11}$. In related studies up to $70 \%$ patients of metabolic syndrome were diabetic. Our results correlate with studies. Most of the patients of metabolic syndrome $(60 \%)$ were asymptomatic i.e. having no symptoms suggestive of hepatic involvement. They were diagnosed as a case of NAFLD on the basis of abnormal liver functions tests, ultrasonographic abnormalities and histopathological changes. When symptoms occur they are non specific like persistent fatigue/malaise $(36.7 \%)$, loss of appetite $(26.7 \%)$ 
and right upper quadrant discomfort (20\%). Mafrad PS. et al $^{\mathbf{1 2}}$ reported above symptoms in about $40 \%-60 \%$ of patients.

Amarapurkar D. et al ${ }^{\mathbf{1 3}}$ observed that mean AST and ALT level were 40.2 $\pm 17.1 \mathrm{U} / \mathrm{L}$ and $44.4 \pm 21.7$ $\mathrm{U} / \mathrm{L}$ respectively. Alanine amniotransferase level had no correlation with histological severity of steatohepattitis. $33.3 \%$ patients of metabolic syndrome had increased hepatic echogenicity on USG abdomen, while $66.7 \%$ patients had normal sonographic studies. Only $53.3 \%$ patients of metabolic syndrome with abnormal liver function tests had increased hepatic echogenicity.

However a study with large number of patients may be needful to prove the above conclusions.

\section{References}

1. Kylin E. Studien uber das HypertonieHyperglyka 'mie-Hyperurika' miesyndrom Zentralbl Inn Med 1923;44:105-127.

2. Kaplan NM. The deadly quartet. Upperbody obesity, glucose intolerance, hypertiglyceridemia, and hypertension. Arch Intern Med 1989; 149:1514-1520.

3. Resnic HE, Jones $\mathrm{K}$, Ruotolo $\mathrm{G}$ et al. Insulin resistance, the metabolic syndrome and risk of incidence cardiovascular disease in non-diabetic American Indians: The Strong Heart Study. Diabetes Care 2003;26:861-867.

4. Resnick HE, Jone K, Ruotolo $G$ et al., Insulin resistance, the metabolic syndrome and risk of incidence cardiovascular disease in nondiabetic American Indian: The strong Heart Stage Diabetes Care 2003;26:861-867.

5. Grundy SM, Brewer HB, Cleeman JI et al. Definition of metabolic syndrome: report of the National Heart, Lung, and Blood Institute/American Heart Association conference on scientific issues related to definition. Circulation 2004;109:433-438.
6. Alberti, K.G., Zimmet, P., Shaw J. for the IDF epidemiology Task force consensus Group 2005. The metabolic syndrome a new world wide definition. Lancet, 366, 1059-62.

7. NCEP .Expert panel on detection, evaluation and treatment of high blood pressure in adult. Executive summary of third report of the National Cholesterol Education Progam (NCEP) expert panel on detection and evaluation and treatment of high blood cholesterol in adult. (Adult Treatment Panel III).JAMA 2001;285:2486-2497.

8. Burnt EM et al. Non-alcoholic steatohepatitis: A proposal for grading and staging the histological lesians. Am J Gastroenterol 1999; 94 (9): 2467-74.

9. Angulo P, Lindor KD. The natural history of non-alcoholic fatty liver disease: a population based cohort study. Gastroenterology Hepatology 2002; 17 (suppl).

10. Diehl AM, Goodman Z, Ishak KG. Alcoholic liver disease in nonalcoholic: a clinical and histologic comparison alcoholinduced liver disease. Gastroenterology 1988;95:1056-62.

11. Flack-Yatter Y, Mc Cullough AJ, Younossi ZM. Clinical feature and natural history of nonalcoholic steatosis Semin Liver Dis 2001; 21:17-26.

12. Mofrad PS, Sanyal AJ. Non-alcoholic fatty liver disease. Medsoape General Medicine 2003;5 (2).

13. Amarapurkar DN, Pater ND, Gupte P et al. Nonalcoholic Steatohepatitis (NASH) diabetes predictor of liver fibrosis. 